\title{
Prevalence and Antimicrobial Resistance Profile of Listeria monocytogenes from Retailed Fresh Cat Fish and Frozen Fish in Abuja, Nigeria.
}

\author{
Alu, J. A ${ }^{1 *}$., Enem, I. S ${ }^{1}$, Nafarnda, W.D ${ }^{1}$., Omeiza, K. G ${ }^{1}$, and Olabode, H. O. K. ${ }^{2}$ \\ ${ }^{I}$ Department of Veterinary Public Health and Preventive Medicine, Faculty of Veterinary Medicine, \\ University of Abuja, F.C.T., Nigeria. P.M.B. 117, Abuja, Nigeria. \\ ${ }^{2}$ Department of Veterinary Microbiology, Faculty of Veterinary Medicine, \\ University of Abuja, F.C.T., Nigeria. P.M.B. 117, Abuja, Nigeria.
}

Accepted June, 2021 and Published July, 2021

\begin{abstract}
This study investigated the prevalence and antibiotic resistance profile of Listeria monocytogenes from fresh catfish (Clarias species) and frozen raw fish (Mackerel and Herring). A total of 180 samples which comprised 60 units of fresh catfish, mackerel, and herring frozen fish each distributed uniformly across three locations in Abuja, were randomly collected within a period of three months. The samples were analyzed using standard bacteriological methods at the Animal science/bacteriology laboratory, Faculty of Agriculture, University of Abuja. Presumptive isolates were further serotyped using latex agglutination and subjected to antimicrobial sensitivity testing at the Advance Biotechnology laboratory (SHESTCO) Sheda, Abuja. Overall prevalence of L. monocytogenes in this study was 10.6\% $(n=19 / 180)$ distributed across Gwagwalada (20\%), Bwari (11.7\%) and none in Kwali Area council. The occurrence according to fish types showed $18.3 \%$ in mackerel, $10.0 \%$ in herring fish while $3.33 \%$ was from catfish. There was no statistically significant difference $(p>0.05)$ between the prevalence of Listeria monocytogenes in the fish type studied. Antimicrobial resistance profile indicated high MAR index $(\geq 0.4)$ which revealed a diverse spread of bacterial resistance to antibiotics within the fish population in the study area. This was characterized by $100 \%$ resistance of L. monocytogenes isolates to ampicillin and tetracycline while few (5) isolates were susceptible to chloramphenicol (71.5\%), streptomycin $(61.2 \%)$. This finding provides a baseline information on the prevalence and antibiotic profile of $L$. monocytogenes in catfish and frozen fish in Abuja, Nigeria. Proper hygienic handling of fish during processing and sales is optimum in mitigating the risk of foodborne illness due to L. monocytogenes. Adequate control of antimicrobial agent usage is also recommended to reduce the occurrence and spread of potential multidrug resistance strains.
\end{abstract}

Keywords: L. monocytogenes, Antibiotic-resistance, Serotyping, Latex agglutination, Fish

\footnotetext{
*Corresponding author:

email: adaeze.alu@uniabuja.edu.ng

Tel: +234-803 4377070
} 


\section{INTRODUCTION}

Bacteria genus Listeria are gram-positive facultative anaerobic, non-spore forming rods, food-borne pathogen, currently contains six species, namely $L$. monocytogenes, $L$. innocua, L. welshimeri, L. seeligeri, L. ivanovii and $L$. grayi and four sub-species. This zoonotic organism was discovered around 100 years ago, Listeria are commonly found in the natural environment, four species have been implicated in cases of human illness but L. monocytogenes is by far the most important due to its virulent nature and implication in most cases of human infection [1]. All species can cause listeriosis, $L$. monocytogenes can cause severe life threatening infections in pregnant women, newborn babies, the elderly with immunocompromised being the most vulnerable group at risk $[1,2]$.

Listeria can be found in different types of food of plant and animal origin. Transmission of infection is mainly foodborne characterized by serious public health problems, however, person-to-person transmission are rare [3]. Up until the 1980's, Listeria was considered a rather rare and little known bacterial genus, the notion began to change in 1981, when it was first discovered that $L$. monocytogenes could be transmitted by contaminated food. In 1980's, North America and Europe reported several foodborne outbreaks of listeriosis, the rate of the disease incidence rose steadily year by year. Various outbreaks led the World Health Organization to conclude that $L$. monocytogenes was an environmental contaminant mainly transmitted through food to humans [4]. In recent years, about 1600 cases of listeriosis were recorded in the USA annually, with approximately 260 deaths. Also about 1470 cases were recorded in 2011 and 864 cases in 2018 respectively, which indicated a continuous incidence especially in the elderly [5]. L. monocytogenes is ubiquitous in the environment and can be isolated from soil, plant materials animals and even marine fish and sea food. It has also been found in a very wide variety of food products, but it can be present as virulent bacterial in chilled processed foods with a long shelf life, such as smoked fish, soft cheeses and ready to eat cooked meats, possibly due to its ability to grow slowly at temperatures as low as $0^{\circ} \mathrm{C}$ and multiplication to dangerous state in refrigerated foods unless controlled [6].

Epidemiological data also indicated, minimal infectious dose of Listeria for listeriosis is at least $100 \mathrm{CFU} / \mathrm{g}$ of food [7, 8]. However, prolonged daily consumption of food containing about $10^{1}-10^{5} \mathrm{CFU} / \mathrm{g}$ of $L$. monocytogenes can result to infections due to Listeria [9]. This have been isolated from various fish types that had posed great danger to human health.

Fish are the cheapest source of animal protein in Africa [10], which have high water content and freezes between temperatures of 0 and $3^{\circ} \mathrm{C}$ with an average of about $2^{\circ} \mathrm{C}$ [11]. Freezing destroys most bacteria but upon thawing, survivals rapidly regain their growth [12]. Fish has been recognized as a vehicle for the transmission of L. monocytogenes [13], research findings suggested that consumers were exposed to L. monocytogenes through consumption/ ingestion of traditionally processed fish from informal markets [14].

In Nigeria, fish are good sources of protein and major staple in various delicacies and has contributed significantly as source of food for the increasing population, currently estimated at 186 million people by the World Bank [15]. Nigeria was also ranked as the third most dependent population globally on coastal fisheries for food and nutrition security, characterized by a growing demand for fish, alongside growth in population and incomes. However, there exist a low household fish consumption measured at $13.3 \mathrm{~kg} /$ capita/year when compared with the world's average of $20.3 \mathrm{~kg} / \mathrm{capita} /$ year [16]. Although, Nigeria produces around 1 million tons of fish per year: over 750,000 tons from capture fisheries and roughly 310,000 tons from aquaculture [17]. Commonly consumed species of fish include crayfish, sardines (freshwater and saltwater), 
frozen fish mackerel, herring (Shawa), as well as cultured fish species, such as tilapia, carp and catfish. Unwholesome fish sourced from informal markets in developing countries like Nigeria has great degree of health hazard to its consumer, especially when implicated with $L$. monocytogenes [14]. Hence, the dearth of information on this bacteria in fish within Abuja, prompted this study on the prevalence and antimicrobial profile of $L$. monocytogenes from fish in Abuja, Nigeria, to highlight the potential public health risk for listeriosis and proffer suitable preventive and control plan.

\section{MATERIALSAND METHODS}

\section{Study Area:}

This study was carried out in Abuja, Federal Capital Territory (FCT), which is made up of six Area councils namely, Abaji, Kwali, Gwagwalada, Kuje, Bwari and Abuja Municipal Council (AMAC). Geographically, F.C.T. is placed at the latitude 9.0578499 and longitude 7.49508 , in the northern hemisphere. Sharing borders with Niger state to the west/north, Kaduna state to the north/east, Nasarawa state to the east/south and Kogi state to the south/west, covering a landmass of approximately $7,315 \mathrm{~km}^{2}$, have moderate climatic conditions, with the estimated population of $1,406,239$ as at the 2006 population census [18].

\section{Sample Collection:}

Random sampling method was used to collect the total of 180 fish samples for this study. Samples collected were mackerel fish $(n=60)$, herring $(n=60)$ and catfish $(n=60)$ from local markets randomly selected in three area councils of F.C.T., namely; Bwari, Gwagwalada, and Kwali Area Councils. All fish samples were bought and wrapped as they would normally be sold to the consumer from the various markets and were appropriately labeled, then placed in a flask with ice and transported immediately for dissections and processing within 2 to 6 hours post- collection at the Animal science/bacteriology laboratory, Faculty of Agriculture, University of Abuja. Extracted fish gills, skin and muscles were the principal tissues processed and used for standard bacteriological evaluations. Presumptive isolates were stored and transported for further investigation (serotyping using latex agglutination and antimicrobial sensitivity testing) at the Advance Biotechnology laboratory, Science and Technology Complex (SHESTCO), Sheda, Abuja.

\section{Bacteriological Examination:}

The stomached samples were weighed and $1 \mathrm{gm}$ was inoculated into $10 \mathrm{ml}$ of pre-enrichment culture medium of the Buffered Listeria Enrichment Broth (BLEB) and incubated at $30^{\circ} \mathrm{C}$ for 24 hours. Then $1 \mathrm{ml}$ of the above broth culture was inoculated into pre-enrichment media containing selective agents (Oxoid Novel Enrichment-ONE) to inhibit the growth of competing microorganisms and incubated for additional 24 hours at $35-37^{\circ} \mathrm{C}$. The selective enrichment culture was subsequently inoculated on another selective agar media, [Oxiod Chromogenic Listeria Agar - OCLA prepared with OCLA selective supplement (SRO226)] which demonstrated the utilization of aesculin as aesculin hydrolysis by the Listeria spp., characterized by distinct colonies post incubation at $37^{\circ} \mathrm{C}$ for $24-48$ hours. These typical colonies were then counted as presumptive Listeria colonies and identified morphologically post-Gram staining, which were further confirmed using biochemical and Latex Agglutination tests.

Biochemical tests on the presumptive Listeria colonies were performed and the isolates were identified according to standard methods [19], and then stored in the refrigerator at $4^{\circ} \mathrm{C}$ on slants of nutrient agar for further work.

\section{Serotyping of the Isolates:}

Presumptive Listeria colonies were characterized using Oxoid Listeria rapid test 
method based on antibodies specific to Listeria, to detect L. monocytogenes. The serotyping was done by slide agglutination using antisera of rapid latex agglutination test kit (Oxoid). Drops of antisera were placed on slide trays with wells, smears of colonies were then added to the antisera and mixed thoroughly, and the mixture were examined for reactive agglutination. The isolates were tested using the control latex reagents for nonspecific agglutination of organisms with latex. Strains that agglutinate with latex reagents were considered as Listeria spp and L. monocytogenes serotypes $[19,20]$.

\section{Antibiotic Resistance testing:}

Antimicrobial susceptibility testing of the Listeria isolates to different antimicrobial agents was conducted to establish resistance and calculate the MAR index in accordance to the Clinical and laboratory standards institute guidelines and agar disk diffusion method [21]. This procedure employed the use of 12 commercially available antimicrobial agents (Oxiod) on Mueller-hinton agar (USA), which include ciprofloxacin (CIP) $5 \mu \mathrm{g}$, erythromycin (E) $15 \mu \mathrm{g}$, ampicillin (AMP) $10 \mu \mathrm{g}$, cefoxitin (FOX) $30 \mu \mathrm{g}$, amoxicillin/calvulanic acid (AMC) $30 \mu \mathrm{g}$, sulphamethoxazole/ trimethoprim (SXT) $25 \mu \mathrm{g}$, tetracycline (TE) $30 \mu \mathrm{g}$, amikacin (AK) $30 \mu \mathrm{g}$, streptomycin (S) $10 \mu \mathrm{g}$, nitrofurantoin (F) $300 \mu \mathrm{g}$, chloramphenicol (C) $30 \mu \mathrm{g}$, and gentamicin (CN) $10 \mu \mathrm{g}$. The agar plates were prepared in accordance with standard methods outlined in the manufacturer guidelines. Using, a sterile glass spreader, broth culture was spread gently over the surface of agar plates and allowed to dry for $5 \mathrm{~min}$. The antibiotic discs were firmly placed on the agar surface with $1 \mathrm{~cm}$ distance apart and incubated at $37^{\circ} \mathrm{C}$ for 20 hours. The diameter of inhibition zone formed around each disc was measured using a millimetre ruler and evaluated according to CLSI 2020 [21]. Multiple Antibiotics Resistance (MAR) Index was determined using the formula MAR $=\mathrm{a} / \mathrm{b}$ as described by [22]. Where; $\mathrm{a}=$ the number of antibiotics to which the bacterial isolate displayed resistant. $\mathrm{b}=$ the total number of antibiotics tested.

\section{Statistical Analysis}

The number of positive isolates were expressed as simple descriptive statistics (frequency, percentage and bar charts). Chi square analysis (SPSS: version 20.0) was used to determine significant differences among the different variables (types of fish and locations) at $95 \%$ C.I. Values less than $0.05(\mathrm{P}<0.05)$ were considered significant.

\section{RESULTS}

Cultural characteristics of typical colonies of Listeria spp. (1 $\mathrm{mm})$ grown on Oxiod chromogenic Listeria agar, Post 24 hours incubation indicated grayish colonies surrounded by black halos. After 48 hours colonies became darker, with possible greenish sheen, which were about $2 \mathrm{~mm}$ in diameter, with black halos and sunken centers (Figure 1). Morphological identification post Gram staining demonstrated Gram positive rods while, biochemical characterization test of presumptive Listeria isolates, showed catalase positive, fermentation of sugars indicated as Dglucose positive, mannitol positive, xylose positive, and rhamnose negative (Table 1).

The overall prevalence of $L$. monocytogenes in all the fish studied was $10.6 \%(n=19 / 180)$ from all the Area councils sampled. Prevalence of $18.3 \%(n=11 / 60)$ from mackerel fish consisting of 6 from gills, 3 from skin, and 2 from muscles, while prevalence of $10.0 \%(n=6 / 60)$ from herring fish consisting of 3 from gills, 2 from skin and 1 from muscles, and prevalence of $3.33 \%(n=2 / 60)$ from catfish, only from muscles. There was no statistically significant difference $(p>0.05)$ observed between the prevalence of Listeria in the fish studied. (Table 2).

The antimicrobial testing showed resistance of all the L. monocytogenes isolates to ampicillin $(19 / 19,100 \%)$ and tetracycline $(19 / 19,100 \%)$. While some isolate were resistant to 
sulphamethaxazole/trimethoprim (18/19, five (5) isolates were susceptible to $94.7 \%)$, ciprofloxacin $(16 / 19,84.2 \%)$, cefoxitin chloramphenicol $(71.5 \%)$, and streptomycin $(16 / 19,84.2 \%)$, amikacin $(16 / 19,84.2 \%), \quad(61.2 \%)$ as showed in Table3 and Figure 2. The erythromycin $(14 / 19,73.7 \%)$, nitrofurantoin calculated Multiple antibiotics resistance $(13 / 19,68.4 \%)$, amoxicilin clavulanic acid (MAR) index in this study was MAR Index $\geq$ $(12 / 19,63.2 \%)$, gentamycin $(12 / 19,63.2 \%) \quad 0.4=100 \%$ Listeria Isolates.

and chloramphenicol $(11 / 19,57.9 \%)$. However,

Table 1: Biochemical characteristics of Presumptive Listeria isolates in fresh and frozen fish

\begin{tabular}{|c|c|c|c|c|c|c|c|}
\hline \multirow[b]{2}{*}{$\begin{array}{l}\text { Gram } \\
\text { reaction } \\
\text {, Shape }\end{array}$} & \multirow[b]{2}{*}{$\begin{array}{l}\text { Catalase } \\
\text { reaction }\end{array}$} & \multicolumn{5}{|c|}{ Sugar Fermentation } & \multirow[b]{2}{*}{$\begin{array}{l}\text { Confirmed } \\
\text { organism }\end{array}$} \\
\hline & & $\begin{array}{l}\text { D- } \\
\text { Glucose }\end{array}$ & Mannitol & Xylose & Rhamnose & $\begin{array}{l}\text { Presumptive } \\
\text { organism }\end{array}$ & \\
\hline,+ rods & + & + & + & + & - & L. monocytogenes & $\begin{array}{l}L . \\
\text { monocytogenes }\end{array}$ \\
\hline,+ rods & + & + & + & - & - & Listeria spp. & \\
\hline
\end{tabular}

Table 2: Prevalence of Listeria monocytogenes in different fish types in Abuja -Nigeria.

\begin{tabular}{lllll}
\hline Locations & $\begin{array}{l}\text { Sample } \\
\text { (Fish Type) }\end{array}$ & \multicolumn{3}{l}{ L. monocytogenes } \\
& & - ve (\%) & + ve (\%) & Total No \\
\hline \multirow{4}{*}{ Gwagwalada } & Mackerel Gill & 14 & $6(30.0)$ & 20 \\
& Mackerel Skin & 17 & $3(15.0)$ & 20 \\
& Mackerel Muscles & 18 & $2(10.0)$ & 20 \\
Bwari & Herring Gill & 17 & $3(15.0)$ & 20 \\
& Herring Skin & 18 & $2(10.0)$ & 20 \\
& Herring Muscles & 19 & $1(5.0)$ & 20 \\
\multirow{4}{*}{ Kwali } & Catfish Gill & 20 & - & 20 \\
& Catfish Skin & 18 & $2(10.0)$ & 20 \\
& Catfish Muscles & 20 & - & 20 \\
& & & & \\
\hline
\end{tabular}


Table 3: Antibiotic resistance patterns of Listeria monocytogenes in fresh and frozen fish

\begin{tabular}{|c|c|c|c|c|c|c|c|c|}
\hline \multirow{2}{*}{$\begin{array}{l}\text { Antibiotics } \\
\text { Ampicillin - (Amp) } 10 \mu \mathrm{g}\end{array}$} & \multicolumn{2}{|c|}{ Total Listeria } & \multicolumn{2}{|c|}{ Mackerel fish } & \multicolumn{2}{|c|}{ Herring fish } & \multicolumn{2}{|c|}{ Catfish } \\
\hline & $19 / 19$ & $100 \%$ & $11 / 11$ & $100 \%$ & $6 / 6$ & $100 \%$ & $2 / 2$ & $100 \%$ \\
\hline Tetracycline - (TE) $30 \mu \mathrm{g}$ & $19 / 19$ & $100 \%$ & $11 / 11$ & $100 \%$ & $6 / 6$ & $100 \%$ & $2 / 2$ & $100 \%$ \\
\hline $\begin{array}{l}\text { Sulphamethoxazole/trimethoprim } \\
\text { (SXT) } 25 \mu \mathrm{g}\end{array}$ & $18 / 19$ & $94.7 \%$ & $10 / 11$ & $90.9 \%$ & $6 / 6$ & $100 \%$ & $2 / 2$ & $100 \%$ \\
\hline Ciprofloxacin (CIP) $5 \mu \mathrm{g}$ & $16 / 19$ & $84.2 \%$ & $10 / 11$ & $90.9 \%$ & $5 / 6$ & $83.3 \%$ & $1 / 2$ & $50 \%$ \\
\hline Cefoxitin (FOX) $30 \mu \mathrm{g}$ & $16 / 19$ & $84.2 \%$ & $11 / 11$ & $100 \%$ & $3 / 6$ & $50.0 \%$ & $2 / 2$ & $100 \%$ \\
\hline Amikacin (AK) $30 \mu \mathrm{g}$ & $16 / 19$ & $84.2 \%$ & $10 / 11$ & $90.9 \%$ & $4 / 6$ & $66.7 \%$ & $2 / 2$ & $100 \%$ \\
\hline Erythromycin (E) $15 \mu \mathrm{g}$ & $14 / 19$ & $73.7 \%$ & $9 / 11$ & $81.8 \%$ & $4 / 6$ & $66.7 \%$ & $1 / 2$ & $50 \%$ \\
\hline nitrofurantoin (F) $300 \mu \mathrm{g}$ & $13 / 19$ & $68.4 \%$ & $8 / 11$ & $72.7 \%$ & $5 / 6$ & $83.3 \%$ & $0 / 2$ & $0 \%$ \\
\hline Amoxicillin/calvulanicacid(AMC)30 $\mu \mathrm{g}$ & $12 / 19$ & $63.2 \%$ & $9 / 11$ & $81.8 \%$ & $2 / 6$ & $33.3 \%$ & $1 / 2$ & $50 \%$ \\
\hline Gentamicin $(\mathrm{CN}) 10 \mu \mathrm{g}$ & $11 / 19$ & $57.9 \%$ & $6 / 11$ & $54.5 \%$ & $3 / 6$ & $50.0 \%$ & $1 / 2$ & $50 \%$ \\
\hline Streptomycin (S) $10 \mu \mathrm{g}$ & $6 / 19$ & $38.8 \%$ & $5 / 11$ & $45.5 \%$ & $1 / 6$ & $16.7 \%$ & $0 / 2$ & $0 \%$ \\
\hline Chloramphenicol (C) $30 \mu \mathrm{g}$ & $5 / 19$ & $28.5 \%$ & $3 / 11$ & $27.3 \%$ & $2 / 6$ & $33.3 \%$ & $0 / 2$ & $0 \%$ \\
\hline
\end{tabular}

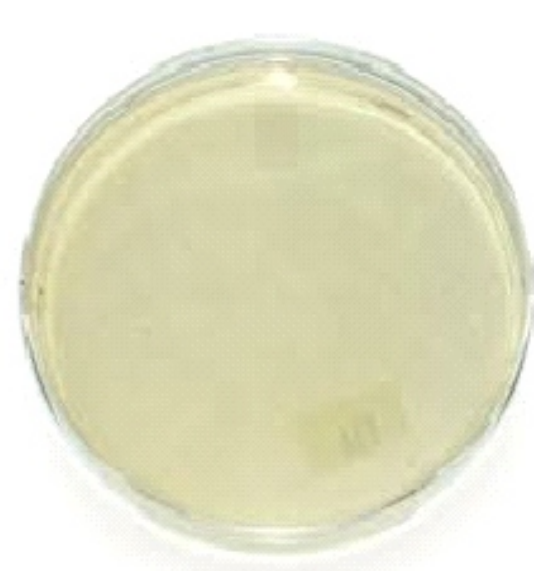

Control



Listeria spp.

Figure 1: Colonial morphorlogy ofListeria spp. on Oxiod Chromogenic Listeria Agar from fish sample in Abuja-Nigeria. 


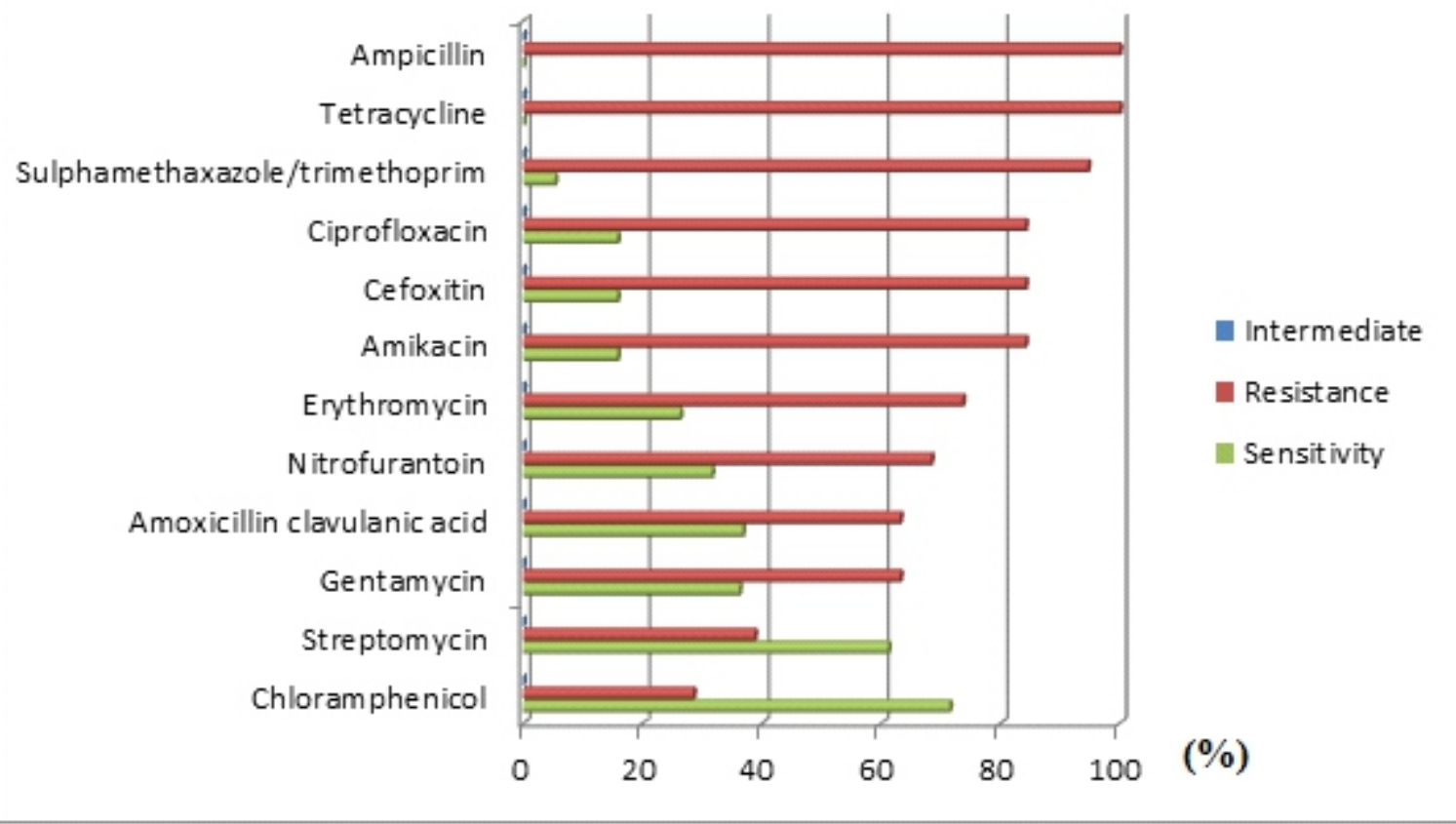

Figure 2: Antibiotic resistance profile of L. monocytogenes isolates from fresh an d frozen fish in Abuja -Nigeria.

\section{DISCUSSION}

In this study, highest occurrence of this organism was observed in the frozen fish mostly mackerel fish followed by herring fish and the least occurrence in catfish, from Gwagwalada and Bwari Area councils. The result of this study agrees with the work done by Amusan [23], whose report indicates $27 \%$ prevalence of $L$. monocytogenes from frozen fish in Lagos, mostly in the mackerel (titus) fish. Similarly, studies of Abdellrazeq et al. [24], also indicates occurrence of Listeria species from fish with an overall prevalence of $56.9 \%$ and $0.96 \%$ mainly from mackerel and catfish, corroborating the findings from this study as shown by the high prevalence in mackerel and low prevalence in catfish. The low prevalence of L. monocytogenes on catfish skin in this present study could be in line with reports of Bang-Yuan et al. [25], which indicates their inability to isolate $L$. monocytogenes strains from catfish skins and intestines but on fish contact surfaces during processing with a frequency of $76.7 \%$ in chilled fresh catfish fillets and $43.3 \%$ in unchilled fillets suggesting that L. monocytogenes in the processed catfish fillets originates from contamination by the processing environment rather than directly from catfish. This however, contrast reports of Chukwu et al. [26] that demonstrates isolation of Listeria species in retailed smoked catfish (Clarias species) in Jos, with a $L$. monocytogenes prevalence of $9.2 \%$ as this further corroborates the demonstration of $L i s t e r i a$ species and especially $L$. monocytogenes in both fresh and frozen fish in Abuja and by extension North-Central Nigeria.

The antibiotic resistance of $L$. monocytogenes in this study agrees with the work of Abdellrazeq et al. [24], whose $L$. monocytogenes isolates were resistance to ampicillin (100\%) and tetracycline (100\%). In addition, reports from work done by Ennaji et al. [27] indicates L. monocytogenes were also 
resistant to nalidixic acid (100\%), cefotaxime $(100 \%)$, cephalothin $(10 \%)$ and susceptible to ampicillin, gentamicin and chlororamphenicol. Similarly, Soni et al. [28], reports that all $L$. monocytogenes isolates except two, were resistance to ampicillin while showing variable resistance to gentamicin, cotrimoxazole, ofloxacin, rifampcin and tetracycline. In contrast, Ruiz-Bolivar et al. [29] reports indicates the susceptibility of $L$. monocytogenes as 100\% for ampicillin, amoxicillin/clavulanic acid, vancomycin and chloramphenicol, whereas for sulphamethaxazole/trimethoprim (98\%), penicillin (97.8\%), azithromycin(96\%), erythromycin $(91 \%)$, ciprofloxacin $(84.4 \%)$, tetracycline $(82 \%)$, meropenem $(71.1 \%)$, rifampin $(64.4 \%)$, and clindamycin $(22.2 \%)$. These varied reports provides evidence for diverse antibiotic resistance patterns in different regions but characterized by an increasing trajectory of resistance of $L$. monocytogenes to antibiotics globally. The MAR index $\geq 0.4$ also and further suggests high risk of contamination and bacterial resistance in this study area as antibiotics are possibly being misuse by fish farmers and environmental waste. This undesired consequences of multidrug resistance, may lead to a cyclic occurrence and spread of multidrug resistance strains post genetic recombination in pollutant environment. Hence, this would require further elucidation as it was not within the current study scope. However, overall prevalence of $10.6 \%$ of $L$. monocytogenes and high MAR Index further indicates presence of a serious potential public health pathogen (Listeria) contaminated with frozen fish and fresh cat fish in the study area. This could lead a fulminating outbreak if uncontrolled.

\section{Conclusion}

This investigation reveals an overall prevalence of $10.6 \%$ of $L$. monocytogenes in frozen fish and fresh cat fish in the study area characterized by high MAR Index and antibiotic resistance especially to Ampicillin and Tetracycline. The result further contributes a valuable baseline information on the prevalence of L. monocytogenes in fish in Abuja, Nigeria.

\section{Recommendation}

Advocacy on proper handling and adequate hygienic measures during fish processing and sales is required to optimally mitigate the risk of contamination due to L. monocytogenes in fish. Control measures in the use of antimicrobial agents is recommended to reduce the spread of undesired consequences of multidrug resistance.

\section{Acknowledgement}

Authors acknowledge the staff of Animal science laboratory, University of Abuja and Advance Biotechnology laboratory, Science and Technology complex (SHESTCO), Sheda, Abuja, for creating good enabling research environment during the period of this work.

\section{Conflicts of Interests}

There are no conflicts of interest between the authors regarding the publication of this paper. 


\section{REFERENCE}

1 Rocourt J., Hof H., Schrettenbrunner A. Malinvereni R., and Bille, J. (1986). Acute purulent Listeria seelingeni meningitis in an immunocompetent adult. Schweiz.Med. Wochenschr. 116:248-251.

2 Cummins A.J., Fielding A. K. and McLauchlin J. (1994). Listeria ivanovii infection in a patient with Aids. Journal of infection. 28:89-91.

3 BfR Federal Institute for Risk Assessment (2020). "Invisible threat: Listeria in smoked fish." Science Daily, $7 \quad 0 \mathrm{c} \mathrm{t} \quad 2020$. www.sciencedaily.com/releases/2020/ 10/201007123057.htm.

4 Montville, T.J. and Matthews, K.R. (2005). Food Microbiology: An Introduction. ASM Press, Washington D.C. pp 159-171.

5 Centers for Disease Control and Prevention, (2019). Listeria (Listeriosis) Outbreak of Listeria Infection. www.cdc.gov.

6 Jay, J.M., Loessner, M.J. and Golden, D.A. (2005). Modern food microbiology. 7th ed. Springer Science+Business Media, Inc. p591.

7 Ooi, S.T., and Lorber, B. (2005). Gastroenteritis due to Listeria monocytogenes. Clinical Infectious Diseases, 40:1327-1332.

8 Yang, H., Qu, L., Wimbrow, A.N., Jiang, X., and Sun,Y., (2007). Rapid detection of Listeria monocytogenes by nanoparticle-based immune-magnetic separation and real-time PCR. International Journal Food of Microbiology. 118:132-138.

9 Maijala, R., Lyytikäinen, O., Johansson, T., Autio, T., Aalto, T., Haavisto, L., and Honkanen-Buzalski T., (2001). Exposure of Listeria monocytogenes within an epidemic caused by butter in Finland.
International Journal of Food Microbiology.70:97-109

10 Claucas. I.J. and Ward. A.R. (1996). Post-harvest Fisheries Development: A Guide to Handling. Preservation. Processing and Quality. Charthan Maritime. Kent. United Kingdom.

11 Desrosier, N.W. (1978). The Technology of Food Preservation 3rd Edition, Colombia University Press, Colombia.

12 Frazier, W.C. and Westhoff, D.C. (1988). Food Microbiology. 4th Edition, McGraw Hill.

13 Adu-Gyamfi, A. (2006). Studies on microbiological quality of smoked fish in some markets in Accra, Ghana. Ghana Journal Science. 46: 67-75.

14 Kennedy B.O. (2011). Risk Assessment for Listeria monocytogenes in Traditionally Processed Fish from Informal Markets in Accra and Tema. Published Thesis, University of Ghana. July 2011.

15 World Bank, (2019). Population total. United Nations Population Division, World Population Prospects: 2019 Revision.

16 FAO, (2018). Food and Agriculture Organization of the United Nations FAO. Book, 978-92-5-130562-1. http://www.fao.org/3/i9540en/I9540E N.pdf

17 World Fish, (2017). Fish in food systems in Nigeria: A review. https://hdl.handle.net/20.500.12348/4 210

18 National Population Commission (NPC) (2006). 'Enumerators manual', March, 2006.

19 Uta G, Denise H, and Philip M. (2005). Methods for the Isolation and Identification of Listeria spp and 
Listeria monocytogenes: a review, FEMS Microbiology Reviews. 29: 851875.

20 Curiale M.S, Lepper W and Robison B. (1994). Enzyme-linked Immunoassay for detection of Listeria monocytogenes in dairy products, seafoods and meats: collaborative study. Journal of AOAC International. 77:1472-1489.

21 CLSI (2020). Clinical and Laboratory Standards Institute; Wayne, PA: $30^{\text {th }}$ Edition. 2020. p. S200-S250.

22 Paul, S., Bezbaruah, R. L., Roy, M. K., and Ghosh, A. C. (1997). Multiple antibiotic resistance (MAR) index and its reversion in Pseudomonas aeruginosa. Applied Microbology, 24:169-171.

23 Amusan, E. E. (2012). Detection of Listeria Monocytogenes in Frozen Fish in Lagos, Nigeria. Fish Technology Department. Nigerian Institute for Oceanography and Marine Research. http://aquaticcommons.org/24229/2/7 8_opt.pdf

24 Abdellrazeq, G. S., Kamar, A. M., and El-Houshy, S. M. (2014). Molecular characterization of Listeria species isolated from frozen fish. Alexandria Journal of Veterinary Sciences 40 (1):115. DOI: 10.5455/ajvs. 45443

25 Bang-Yuan Chen, Rajkumar Pyla, TaeJo Kim, Jua L. Silva, and Yean-Sung Jung, (2010). Prevalence and contamination patterns of Listeria monocytogenes in catfish processing environment and fresh fillets. Journal of Food Microbiology. DOI: 10.1016/j.fm.2010.02.007

26 Chukwu C.O., Ehizibolo D.O, Mustapha I.A., Chukwu I.D., Olabode O., and Ogbonna C.I. (2006). Listeria species in retail smoked fish at Jos, $\mathrm{N}$ i g e ri a. AJOL. D O I : 10.4314/apra.v2i4.36349.

27 Ennaji, H., Timinouni, M., Ennaji, M., Hassar, M., and Cohen, N., (2008). Characterization and Antibiotic Susceptibility of Listeria monocytogenes Isolated from Poultry and Red Meat in Morocco, Journal of Infection \& Drug Resistance. 1: 45-50.

28 Soni, K.A., Nannapaneni, R., and Hagens, S. (2009). Reduction of Listeria monocytogeneson the surface of fresh channel catfish fillets by bacteriophage Listeria P 100. Foodborne Pathogens and Disease 4:427-434.

29 Ruiz-Bolivar, Z., CarrascalCamacho, A.K., Neuque-Rico, M.C., Gutiérrez-Triviño, C., Rodríguez-Bocanegra, M.X., PoutouPiñales, R.A., and Mattar, S. (2011). Enterobacterial repetitive intergenicconsensus-polymerase chain reaction (ERIC-PCR) fingerprinting reveals intraserotype variations among circulating Listeria monocytogenes strains. African Journal of Microbiology Research. 5:1586-1598 